\title{
Endocytosis in Trypanosoma cruzi
}

\author{
Narcisa Leal Cunha-e-Silva*, ${ }^{*}$, Celso Sant'Anna ${ }^{2}$, Miria G. Pereira ${ }^{1}$ and Wanderley de Souza ${ }^{1,2}$
}

\author{
${ }^{1}$ Laboratório de Ultraestrutura Celular Hertha Meyer, Instituto de Biofísica Carlos Chagas Filho, Universidade Fede- \\ ral do Rio de Janeiro, Av. Carlos Chagas Filho, 373, bloco G subsolo, Cidade Universitária, Ilha do Fundão, Rio de \\ Janeiro, 21941- 902, Brazil \\ ${ }^{2}$ Diretoria de Programas, Instituto Nacional de Metrologia, Normalização e Qualidade Industrial-INMETRO, Av. Nossa \\ Senhora das Graças, 50, Xerém, Duque de Caxias - Rio de Janeiro, 25250-020, Brazil
}

\begin{abstract}
Endocytic activity is particularly intense in Trypanosoma cruzi epimastigotes, while in amastigotes and trypomastigotes it is untraceable. Cargo molecules enters through the cytostome or flagellar pocket at the parasite anterior region, goes along a branched early endosomal network of tubules and vesicles spread from nuclear periphery to the posterior pole, until delivery to reservosomes, the final compartment. Reservosomes are acid compartments that store protein and lipid cargo and also accumulate lysosomal hydrolases, modulating digestive activity. Although T. cruzi infective forms are unable to uptake molecules, its lysosome related organelles represent a potential targets for anti-parasitic chemotherapy.
\end{abstract}

Keywords: Endocytosis, Trypanosoma cruzi, Reservosome, Cytostome.

\section{ENDOCYTOSIS}

Endocytosis is essential for cell survival and has been intensely investigated in mammalian and yeast cells. Eukaryotic cells, including protozoan parasites, possess a remarkable integrated system to internalize nutrients, which involves a set of specific proteins and lipids that coordinate vesicle budding and fusion with target compartments. In most cells, different ways for macromolecule internalization from the plasma membrane have been identified. Basically, according to particle size ingestion and cytoskeleton participation they can be classified as phagocytosis and pinocytosis. These processes mobilize different cell components and they coexist in the same cell type (see [1] for a recent review). After internalization macromolecules gain access to the endo-lysosomal system, a process involving the sequential participation of different compartments which form the endosomal-lysosomal pathway.

\section{ENDOCYTOSIS IN TRYPANOSOMATIDS}

Trypanosomatids comprise a family of protozoan parasites which are causative agents of highly relevant human, animal and plant diseases. Among them, three parasitic protozoa deserve special attention: (i) Trypanosoma brucei, causing African sleeping sickness, (ii) Leishmania sp, causing leishmaniasis and (iii) Trypanosoma cruzi, responsible for Chagas disease or American Trypanosomiasis. Together these pathogenic parasites infect about 40 millions of people in Africa, South America, Europe, and Asia, according to World Health Organization (WHO).

Trypanosomatids have complex life cycle alternating between invertebrate insect vector and vertebrate host

\footnotetext{
*Address correspondence to this author at the Av. Carlos Chagas Filho, 373, bloco $\mathrm{G}$ subsolo, Cidade Universitária, Ilha do Fundão, Rio de, Janeiro, 21941-902, Brasil; Tel/Fax: 55-21-2562-6593; E-mail: narcisa@biof.ufrj.br
}

animals. During the cell cycle, parasites differentiate into distinct development stages as a consequence of adaptation, which is vital to parasite establishment. Adaptation involves morphological and biochemical changes. The etiologic agent of Chagas disease presents three distinct developmental stages: trypomastigote (infective form), amastigote (intracellular proliferative forms found in the vertebrate host) and epimastigote (replicative form found in insect vector). Endocytic activity was only demonstrated in epimastigotes.

Trypanosomiasis is controlled by clinical treatment or vector control or both. The current available chemotherapy against trypanosomes is not effective and has poor efficiency, high toxicity and severe collateral effects are remarkable. In addition, increasing resistance to trypanocidal drugs have been reported $[2,3]$.

Endocytosis in trypanosomatids is a phenomenon dependent on the cell polarity and is restricted to membrane areas devoid of subpellicular microtubules [4]. In contrast to higher eukaryotes, extracellular molecules reach the internal milieu through only two entry sites: the flagellar pocket or the cytostome/cytopharynx complex $[5,6,7]$. The flagellar pocket corresponds to an extracellular compartment formed by an asymmetrical invagination of the protozoan plasma membrane, from where a flagellum emerges. Flagellar pocket membrane is continuous with the flagellar membrane. Usually, at the point of emergence of the flagellum, the membrane lining the flagellum and the protozoan body establish a close contact so that the flagellar pocket appears as a sac [4]. Endocytic and exocytic events take place at the pocket region. Cytostome/cytopharynx complex is a profound and stable invagination of plasma membrane [9] that can reach nuclear region. It is observed only in epimastigote and amastigote forms of the Schizotrypanum sub-genus, such as Trypanosoma cruzi, Trypanosoma vespertilionis and Trypanosoma dionisii. In the case of T. cruzi, it represents a 
singular structure, being acidified by a P-type- $\mathrm{H}^{+}$-ATPase [10], possessing an exhuberant glycocalix [11] and responsible for $85 \%$ of the protozoan endocytic activity [7].

Here we will describe only basic aspects of the endocytic pathway which takes place in Trypanosoma cruzi.

\section{ENDOCYTOSIS IN TRYPANOSOMA CRUZI}

Vesicles containing cargo macromolecules, such as transferrin or peroxidase, and transport particles, as LDL, bud off from flagellar pocket or from the most posterior portion of the cytostome/cytopharinx system (Fig. 1a-c) [5-8]. In silico analysis revealed the presence of clathrin, adaptin and clathrin self-assembly genes as well as dynamin gene [12]. Immunofluorescence using antibodies against bovine clathrin heavy chain detect the protein expression at the flagellar pocket and Golgi complex. The presence of coated vesicles, possibly containing clathrin, was observed in vesicles budding from the flagellar pocket [12] and located at the transmost region of the Golgi complex $[12,13]$. However, all vesicles observed budding from the bottom of the cytostome/cytopharinx system showed an uncoated aspect [6, $7,12]$.
The endocytic vesicles originated either from the cytostome or from the flagellar pocket fused with early endosomes, the branched-tubular compartments spread along the posterior region of epimastigotes (Fig. 1d). They are acidic structures as demonstrated by acridine orange accumulation [7]. From early endosomes, vesicles carrying cargo budded off and fused with the reservosomes, an unusual type of organelle classified as lysosome-related organelles (LROs) [14]. Reservosomes are morphologically characterized as rounded organelles, with an average diameter of $0.4-0.6 \mu \mathrm{m}$ [15], which may contain small vesicles, internal membranes profiles, an electron dense protein matrix and electron lucent lipid inclusions [16]. Some of these lipid inclusions can be observed as rectangular structures or in a needle shape surrounded by a phospholipid monolayer, or yet as spherical inclusions (Fig. 1d). Reservosomes store a large amount of neutral lipids, such as cholesteryl esters [17], probably due to a high rate of endocytosis of LDL and other lipoproteins. It is well established that $T$. cruzi epimastigotes do not synthesize cholesterol, but they are able to insert this molecule into their membranes. Interestingly, the ABCA1 transporter was suggested by immunocytochemistry

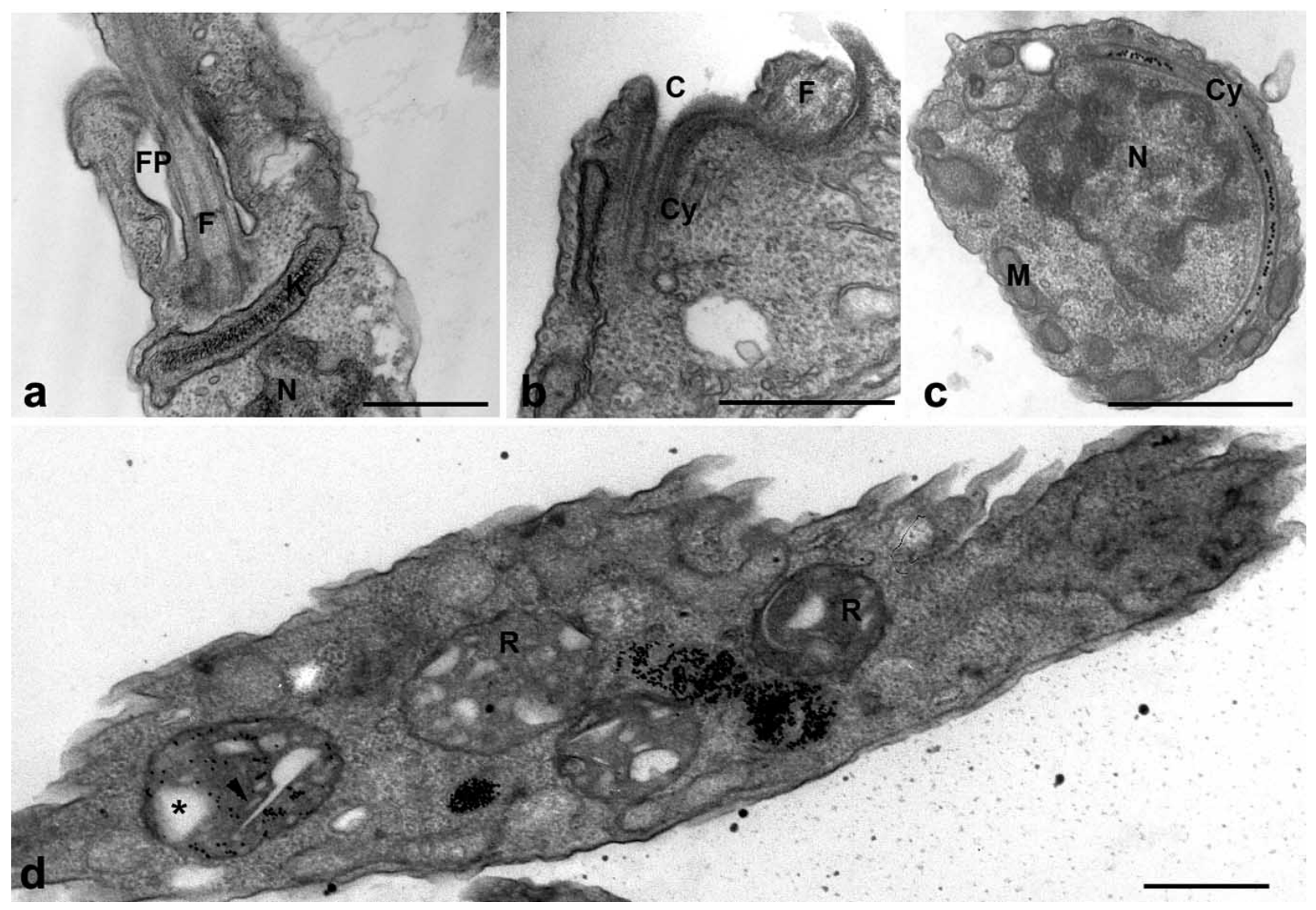

Fig. (1). Trypanosoma cruzi epimastigote main endocytic compartments as observed by transmission electron microscopy of ultrathin sections. a) the anterior region of the parasite body showing the flagellar pocket (FP), at the point of flagellum (F) emergence; b) a longitudinal section of the cytostome opening (C) that continues in the cytopharinx (Cy); note the glycocalix and the cytoskeleton beneath the membrane domain between the cytostome and the flagellar pocket; c) the cytopharinx goes deeply inside the parasite body, and can be observed full of gold labeled cargo in this oblique section at the nuclear region; d) transport vesicles, which may be sections of the branched early endosomal network, concentrate gold labeled cargo that will be delived to reservosomes (R); these big round organelles present their typical aspect: the electron dense matrix containing proteins, including gold-labeled transferrin, and the pleomorphic electron lucent lipid inclusions, which may be round (asterisk) or needle-shaped (arrow). K, kinetoplast; M, mitochondrion. Bars: a) $0.5 \mu \mathrm{m}$; b) $0.5 \mu \mathrm{m}$; c) 0.6 $\mu \mathrm{m}$; d) $0.4 \mu \mathrm{m}$. 
to be localized in reservosomes [18] and the proteomic analysis of the organelle revealed the presence of an $\mathrm{ABC}$ transporter [19]. Moreover, a homologue of Rab 18, TcRab18, was found. In mammals, Rab 18 was localized in lipid bodies and endoplasmic reticulum of many cells types, such as adypocites. This GTPase is involved in the regulation of lipids release to the rest of the cell [20], and probably contributes to lipid distribution from reservosomes to the whole parasite.

The multipurpose character of reservosomes could also be evidenced by the presence of many proteases, such as cruzipain [21], which is endogenously modulated by chagasin [22], serine carboxipeptidase [14, 23], glucosidases, cysteine peptidases, $\alpha$-mannosidases, acid phosphatase and acid phosphatase 2 [19]. All of them function in an acid medium provided by the action of three P-type $\mathrm{H}^{+}$- ATPase isoforms: TcHA 1, TcHA 2 [10] and TcHA 3 [19]. Moreover, enzymes involved in lipid metabolism as lipases, sterol 24-c-methyltransferase or fatty acyl CoA synthetase plus the presence of a multidrug resistance (MDR) protein seem to play an important role in the organelle/cell metabolism. In addition, TcRab 7 and TcRab 11 were identified [24, 25]. TcRab 7 could indicate an intense traffic established between Golgi apparatus and reservosomes, whilst TcRab 11 could be related to recycling process, as described for Rab 11 in mammals [26].

Like in T. brucei lysosomes, reservosomes also present the p67 glycoprotein, which is structurally similar to mammalian LAMPs [20, 27]. While in epimastigotes early endosomes are poorly characterized, T. brucei presents a well defined endocytic route, comprised by early and late endosomes converging protein and lipids uptake into lysosomes (see [28] for a recent review). Although there was some speculation of a lysosome role for reservosomes, they were, indeed, lysosome-related organelles. Recently, a set of vesicles dispersed from anterior to posterior poles of the parasite were suggested as epimastigote lysosomes by the presence of aryl sulphatase [29], but their relationship with reservosomes or their digestive function was not be investigated yet. Reservosomes are also associated with autophagic events, and are particularly important for parasite survival during periods of starvation or differentiation [30].

Recently, organelles that share typical epimastigote reservosome features were identified in trypomastigotes and amastigotes [14]. They are concentrated at the posterior region of the parasite, present cruzipain, and its inhibitor chagasin, serine carboxypeptidase, besides having acid character driven by the action of a P-type proton pump. As well as epimastigote reservosomes, they were classified as lysosome-related organelles (LROs). Their presence in the infective stages of $T$. cruzi may represent a potential target to drug design against Chagas' disease.

\section{ACKNOWLEDGEMENTS}

The authors are grateful to Conselho Nacional de Desenvolvimento Científico e Tecnológico (CNPq), Fundação Carlos Chagas Filho de Amparo à Pesquisa no Estado do Rio de Janeiro (FAPERJ) and to Coordenação de Aperfeiçoamento de Pessoal de Nível Superior (CAPES).

\section{REFERENCES}

[1] Doherty GJ, McMahon HT. Mechanisms of endocytosis. Annu Rev Biochem 2009; 78: 857-902.

[2] Andrade HM, Murta SMF, Chapeaurouge A, et al. Proteomic analysis of Trypanosoma cruzi Resistance to Benznidazole. J Proteome Res 2008; 7: 2357-67.

[3] Urbina JA, Docampo R. Specific chemotherapy of Chagas disease: controversies and advances. Trends Parasitol 2003; 19: 495-501.

[4] De Souza W, Sant'Anna C, Cunha-e-Silva NL. Electron microscopy and cytochemistry analysis of the endocytic pathway of pathogenic protozoa. Prog Histochem Cytochem 2009; 44: 67-124.

[5] De Souza W, Carvalho TU, Benchimol M, Chiari E. Trypanosoma cruzi: ultrastructural, cytochemical and freeze-fracture studies of protein uptake. Exp Parasitol 1978; 45: 101-15.

[6] Soares MJ, de Souza W. Endocytosis of gold-labeled proteins and LDL by Trypanosoma cruzi. Parasitol Res 1991; 77: 461-8.

[7] Porto-Carreiro I, Attias M, Miranda K, De Souza W, Cunha-e-Silva N. Trypanosoma cruzi epimastigote endocytic pathway: cargo enters the cytostome and passes through an early endosomal network before storage in reservosomes. Eur J Cell Biol 2000; 79: 858-69.

[8] Scott DA, Docampo R, Dvorak JA, Shi S, Leapman RD. In situ compositional analysis of acidocalcisomes in Trypanosoma cruzi. J Biol Chem 1997; 272: 28020-9.

[9] Milder R, Deane MP. The cytostome of Trypanosoma cruzi and T. conorhini. J Protozool 1969; 16: 730-7.

[10] Vieira M, Rohloff P, Luo S, Cunha-e-Silva NL, de Souza W, Docampo R. Role for a P-type H+-ATPase in the acidification of the endocytic pathway of Trypanosoma cruzi. Biochem J 2005; 392: 467-74.

[11] Pimenta PF, de Souza W, Souto-Padron T, Pinto da Silva P. The cell surface of Trypanosoma cruzi: a fracture-flip, replica-staining label-fracture survey. Eur J Cell Biol 1989; 50: 263-71.

[12] Correa JR, Atella GC, Menna-Barreto RS, Soares MJ. Clathrin in Trypanosoma cruzi: in silico gene identification, isolation, and localization of protein expression sites. J Eukaryot Microbiol 2007; 54: 297-302.

[13] Sant'Anna C, de Souza W, Cunha-e-Silva N. Biogenesis of the reservosomes of Trypanosoma cruzi. Microsc Microanal 2004; 10: 637-46.

[14] Sant'Anna C, Parussini F, Lourenco D, et al. All Trypanosoma cruzi developmental forms present lysosome-related organelles. Histochem Cell Biol 2008; 130: 1187-98

[15] Soares MJ, De Souza W. Cytoplasmic organelles of trypanosomatids: a cytochemical and stereological study. J Submicrosc Cytol Pathol 1988; 20: 349-61.

[16] Sant'Anna C, Pereira MG, Lemgruber L, de Souza W, Cunha e Silva NL. New insights into the morphology of Trypanosoma cruzi reservosome. Microsc Res Tech 2008; 71: 599-605.

[17] Cunha-e-Silva NL, Atella GC, Porto-Carreiro IA, et al. Isolation and characterization of a reservosome fraction from Trypanosoma cruzi. FEMS Microbiol Lett 2002; 214: 7-12.

[18] Torres C, Perez-Victoria FJ, Parodi-Talice A, Castanys S, Gamarro F. Characterization of an ABCA-like transporter involved in vesicular trafficking in the protozoan parasite Trypanosoma cruzi. Mol Microbiol 2004; 54: 632-46.

[19] Sant'Anna C, Nakayasu ES, Pereira MG, et al. Subcellular proteomics of Trypanosoma cruzi reservosomes. Proteomics 2009; 9:1782-94.

[20] Martin S, Parton RG. Characterization of Rab18, a lipid dropletassociated small GTPase. Methods Enzymol 2008; 438: 109-29.

[21] Souto-Padron T, Campetella OE, Cazzulo JJ, de Souza W. Cysteine proteinase in Trypanosoma cruzi: immunocytochemical localization and involvement in parasite-host cell interaction. J Cell Sci 1990; 96: 485-90.

[22] Santos CC, Sant'Anna C, Terres A, et al. Chagasin, the endogenous cysteine-protease inhibitor of Trypanosoma cruzi, modulates parasite differentiation and invasion of mammalian cells. J Cell Sci 2005; 118: 901-15.

[23] Parussini F, Garcia M, Mucci J, et al. Characterization of a lysosomal serine carboxypeptidase from Trypanosoma cruzi. Mol Biochem Parasitol 2003; 131: 11-23.

[24] Araripe JR, Cunha e Silva NL, Leal ST, de Souza W, Rondinelli E. Trypanosoma cruzi: TcRAB7 protein is localized at the Golgi apparatus in epimastigotes. Biochem Biophys Res Commun 2004; 321: $397-402$. 
[25] Mendonca SM, Nepomuceno da Silva JL, Cunha e-Silva N, de Souza W, Gazos LU. Characterization of a Rab11 homologue in Trypanosoma cruzi. Gene 2000; 243: 179-85.

[26] Zerial M, McBride H. Rab proteins as membrane organizers. Nat Rev Mol Cell Biol 2001; 2:107-17

[27] Alexander DL, Schwartz KJ, Balber AE, Bangs JD. Developmentally regulated trafficking of the lysosomal membrane protein p67 in Trypanosoma brucei. J Cell Sci 2002; 115: 3253-63.

[28] Field M, Carrington M. Intracellular membrane transport systems in Trypanosoma brucei. Traffic 2004; 5: 905-13.
[29] Adade CM, de Castro SL, Soares MJ. Ultrastructural localization of Trypanosoma cruzi lysosomes by aryl sulphatase cytochemistry. Micron 2007; 38: 252-6.

[30] Alvarez VE, Kosec G, Sant Anna C, Turk V, Cazzulo JJ, Turk B. Blocking autophagy to prevent parasite differentiation: a possible new strategy for fighting parasitic infections? Autophagy 2008; 4 : 361-3.

(C) Cunha-e-Silva et al.; Licensee Bentham Open.

This is an open access article licensed under the terms of the Creative Commons Attribution Non-Commercial License (http://creativecommons.org/licenses/by-nc/3.0/) which permits unrestricted, non-commercial use, distribution and reproduction in any medium, provided the work is properly cited. 